- Insbesondere in Bezug auf die Pränataldiagnostik wird neben der Qualität der humangenetischen Beratung auch eine unabhängige (nicht medizinische oder humangenetische) Beratung (zumindest als Option) sowie die Gewährleistung eines entsprechenden institutionalisierten Beratungsangebotes vorgeschrieben.

- Die Einführung genetischer Tests wird nicht dem Markt überlassen. Vielmehr ist eine behördliche Zulassung genetischer Tests vorgesehen.

- Zur Gewährleistung der Kontrolle von gendiagnostischen Einrichtungen, zur Formulierung verbindlicher Standards, für die Zulassung von Tests oder zur Beratung von mit diesen Aufgaben betrauten Behörden wird eine zentrale Kommission eingerichtet, in der Experten verschiedener Disziplinen und verschiedene gesellschaftliche Gruppen vertreten sind.

Der TAB-Bericht wurde am 11. Oktober 2000 im Ausschuss für Bildung, Forschung und Technikfolgenabschätzung zusammen mit dem Bericht der Bundesregierung zum Stand und den Perspektiven der Genomforschung in Deutschland diskutiert. Vertreter aller Fraktionen betonten unter Bezugnahme auf den Bericht des TAB die dringende Notwendigkeit, über gesetzgeberische Maßnahmen zur Regulierung der Testpraxis nachzudenken.

Der TAB-Arbeitsbericht Nr. 66 „Stand und Perspektiven genetischer Diagnostik" kann schriftlich angefordert werden bei:

Büro für Technikfolgen-Abschätzung beim Deutschen Bundestag

Neue Schönhauser Straße 10, D-10178 Berlin

Fax: + 49 (0) 30 / 28491-119

E-Mail: buero@tab.fzk.de

Dr. Leonhard Hennen

Tel.: + 49 (0) 30 / 28491-108

E-Mail: hennen@tab.fzk.de

\section{Herausforderungen an die Technikfolgenforschung durch Liberalisierung - das Beispiel der Strommärkte}

\author{
von Martin Weisheimer, Institut für Wirt- \\ schaftsforschung Halle (IWH)
}

\begin{abstract}
Auf der Fachtagung ,Zehn Jahre TA in den neuen Bundesländern" am 7./8. November 2000 in Chemnitz hat der Vortrag zum oben genannten Problem eine breite Resonanz gefunden. Am Beispiel der liberalisierten Strommärkte (in Deutschland und international) wird demonstriert, welche komplexen technisch-technologischen, ökonomischen, ökologischen und sozialen Folgen mit der Veränderung institutioneller Rahmenbedingungen einhergehen. Da sich der Trend zur Liberalisierung der Wirtschaft - in Einheit mit der Deregulierung und Privatisierung/ Internationalisierung - fortsetzt, handelt es sich um eine zukunftsträchtige Aufgabe der Technikfolgenabschätzung. Sie bietet den Vorzug, den Übergang vom technik- zum probleminduzierten Ansatz zu fördern, weitgehend direkt Unternehmen anzusprechen und im Prozess technischer Entscheidungen recht frühzeitig gesellschaftliche Folgewirkungen zu antizipieren.
\end{abstract}

\section{Charakterisierung des Problems}

Wie in anderen Staaten der Welt vollzieht sich auch in der deutschen Wirtschaft der Trend zur Liberalisierung. Dabei geht - dank der Intensivierung des branchenbezogenen Wettbewerbs die Überwindung des Monopolcharakters mit der Deregulierung sowie Privatisierung und Internationalisierung der Wirtschaft einher. Oft entstehen erstmals Märkte für die Beschaffung und den Absatz. Sie sind für mehrere Anbieter von Gütern und Dienstleistungen offen und ermöglichen dem Verbraucher die freie Auswahl ihrer Lieferanten.

Beispiele für solche geschaffenen liberalisierten (freien geöffneten) Märkte sind bisher die Telekommunikations-, Strom- und Gasbranche sowie das Luftverkehrs- und Eisenbahnwesen, demnächst vermutlich das Postwesen, die Wasserversorgung sowie die Abfall- 
entsorgungswirtschaft. Das heißt, es handelt sich um eine breite Palette von Wirtschaftsbereichen mit bemerkenswerten Leistungs-, Investitions-/Kapital- und Beschäftigungs- sowie sonstigen Potentialen.

Wie weltweit die hierbei gewonnenen praktischen Erfahrungen zeigen, bringen diese neuen institutionellen Rahmenbedingungen gravierende Veränderungen in den jeweiligen Branchen sowie bei den vor- und nachgelagerten Partnern mit sich. In diesen Komplexen bleibt nichts wie es bisher war. Durch den Wettbewerb kommt es in erster Linie zu Rationalisierungen, Modernisierungen und Innovationen sowie zu durchgängigen Preisreduzierungen. Aufgrund der veränderten Markt- und Bewertungsverhältnisse folgen daraus vielfältige, technische/technologische, ökonomische, ökologische und soziale Konsequenzen.

In Deutschland macht das die Liberalisierung der Branchen Telekommunikation (ab Januar 1998) und Stromversorgung (ab April 1999) besonders deutlich. Anhand der deutschen Elektrizitätswirtschaft werden im Folgenden Implikationen und Schlussfolgerungen für die Technikfolgenabschätzung näher behandelt.

\section{2}

\section{Veränderungen auf der Angebotsseite}

Seit anderthalb Jahren werden die deutschen Stromverbraucher nicht mehr von überregionalen, regionalen und kommunalen Monopolen versorgt. Die Elektrizität wird nun von mehreren Lieferanten für alle Kunden auf dem Markt frei zur Auswahl angeboten. Neben den bisherigen Versorgern kommen neue Akteure (unabhängige Erzeuger, Stromhändler, Broker, Importeure etc.) als mögliche Lieferanten in Frage. Außerdem nehmen die Strombörsen (für den Spot- und Terminmarkt) und der InternetEinkauf (e-commerce) an Bedeutung zu.

Allerdings fungieren die überregionalen, regionalen und örtlichen Netze der Stromversorgung nach wie vor als natürliche Monopole. Ihre allgemeine Benutzung zur diskriminierungsfreien Durchleitung von Fremdstrom ist erforderlich, um dennoch den Wettbewerb und die konkurrierende Belieferung bis zum Verbrauchsort zu realisieren. Zur Vermeidung von Interessenkonflikten setzt das zugleich eine buchhalterische bzw. sogar organisatorische
Trennung bisher eng verbundener Geschäftsbereiche (wie Erzeugung, Transport, Verteilung und Verkauf) voraus.

Der Wettbewerb führt zwangsläufig sowohl auf der Angebots- als auch Nachfrageseite $\mathrm{zu}$ grundlegenden Implikationen (vgl. Tab. 1).

Tab. 1: Folgen des Wettbewerbs im Strommarkt

\begin{tabular}{|ll|}
\hline Angebotsseite & Nachfrageseite \\
- Kosteneinsparung/ & - Preissenkungen für \\
Rationalisierung/ & Wirtschaft und Haus- \\
Umstrukturierung & halte \\
- Personaleinsparung/ & - Erschwernis für \\
Qualifikationswandel & Strom-/Energie- \\
- Produktionsreduzie- & einsparungen \\
rung/-stilllegung & - Chancen für Elektro- \\
- neue Technologien/ & technologien, z. B. für \\
Anlagen & Raumwärme \\
- Erhöhung des Strom- & - Akzeptanz für Liefe- \\
imports und -handels & rantenwechsel \\
- Hemmnis für regene- & organisatorische \\
rative Energien & Veränderung, z. B. \\
- Gewinneinbußen im & Bündelung \\
Querverbund & \\
\hline
\end{tabular}

Auf der Angebotsseite kommt es zum Kampf um Kunden und Marktanteile, erst recht, wenn sich die Nachfrage allgemein nur wenig erhöht (in Deutschland maximal um 1,0\%/a) und Überkapazitäten (in Deutschland von etwa $10 \%$ ) bestehen. Stromverkaufsangebote müssen daher insbesondere billiger, besser (mit mehr Beratung und Service) und auf die spezifischen Bedürfnisse zugeschnitten flexibler werden. Durchgängige Rationalisierungen und Kosteneinsparungen in der Erzeugung, Produktionseinstellungen und zugleich neue flexible Produktionsanlagen mit billigeren Brennstoffen und effizienten Technologien/Techniken sind die Folge davon. Konkret reflektiert sich das insbesondere in der Tendenz der drastischen Reduzierung der Personalkosten mit deutlichen Einsparungen bei den herkömmlichen Arbeitsplätzen, des Ausbaus kleiner, billiger und flexibler arbeitenden Erzeugungsanlagen zulasten der bisherigen Großeinheiten, der verstärkten Substitution bisheriger Brennstoffe durch Erdgas sowie des Ausbaus von Kombiprozessen, da die gleichzeitige Erzeugung von Strom und Wärme effizient und umweltverträglich ist. Mithin kommt es zur Orientierung auf dezentrale gas- 
gefeuerte Blockheizkraftwerke (BHKW) und Gas- und Dampfturbinen (GuD)-Anlagen als integrale Lösung.

Diese neue technische/technologische Entwicklungsrichtung passt sich dem nunmehr bestehenden Investitions-, Markt- und Preisrisiko flexibel an. Die spezifischen Anlagenkosten in $\mathrm{DM} / \mathrm{kW}$ liegen weitaus günstiger (etwa bis zur Hälfte), so dass der Zeitbedarf für den wirtschaftlichen Rückfluss der Investitionen wesentlich kürzer (etwa zehn bis zwölf anstatt über zwanzig Jahre) wird. Die Erzeugung kann sich durch die Gasfeuerung und moderne Regelung kurzfristig (etwa in einer Stunde) an den zeitlich schwankenden Strombedarf anpassen. Durch Veränderung des variablen Stromfaktors kann in der Spitzenzeit der Stromanteil erhöht und damit ein wesentlich größerer Verkaufserlös erzielt werden.
Angesichts des eingesetzten Erdgases (anstelle von Kohle, Kernbrennstoff und Öl) sowie der höheren Brennstoffausnutzung und energetischen Wirkungsgrade ergibt sich, dass die Anlagen und Technologien außerdem die Umweltbelastungen (wie $\mathrm{SO}_{2}, \mathrm{CO}_{2}$ und $\mathrm{NO}_{\mathrm{x}^{-}}$ Emission) relevant absenken.

Kurzum: Früher, unter den Bedingungen der Versorgungsmonopole (mit gesichertem Absatz, Kostenpreisen etc.), mussten die Elektrizitätsversorgungsunternehmen (EVU) die Risiken der Entwicklung nicht befürchten. Nunmehr initiiert die Liberalisierung veränderte Rahmenbedingungen, d. h. letztendlich veränderte risikobehaftete Anforderungen an die Art und Weise des Angebots und die hiermit verbundenen ökologischen, ökonomischen und sozialen Folgen (vgl. Tab. 2).

Tab. 2: Konsequenzen der Liberalisierung

\begin{tabular}{|c|c|c|c|}
\hline ökonomische & ökologische & soziale & $\begin{array}{c}\text { organisatorische und } \\
\text { sonstige }\end{array}$ \\
\hline $\begin{array}{ll}\text { - Wettbewerb mit Druck } \\
\text { auf } \\
-\quad \text { Preisniveau } \\
-\quad \text { Gewinn } \\
-\quad \text { Kosten } \\
\text { - } & \text { Absatzmengen } \\
\text { - Veränderte ökonomische } \\
\text { Bewertung von Technik- } \\
\text { varianten wegen } \\
\text { - } \quad \text { Preis-/Kosten-/ } \\
\text { Gewinnentwicklung } \\
\text { getrennte Geschäfts- } \\
\text { bereiche, bessere } \\
\text { Kostenzurechnung, } \\
\text { Kürzung von Quer- } \\
\text { subventionen } \\
\text { - Nachteil hoher Fix- } \\
\text { kostenanteile } \\
\text { - Vorteil flexibler Re- } \\
\text { gime } \\
\text { neue Technikangebote } \\
\text { wegen } \\
\text { - } \\
\text { erweitertem } \\
\text { „Vergleichsmarkt“ } \\
\text { (mit realen und po- } \\
\text { tenziellen Importen) } \\
\text { Internationalisie- } \\
\text { rung/Privatisierung }\end{array}$ & $\begin{array}{l}\text { - Umweltschutz als } \\
\text { Belastung und } \\
\text { Rationalisierungsdruck } \\
\text { - } \text { Bevorzugung billiger } \\
\text { Brennstoffe und Technik } \\
\text { - } \quad \text { Erdgas vs. Kohlen, } \\
\text { Kernenergie } \\
\text { - Kessel, Turbinen, } \\
\text { Generatoren, } \\
\text { Leitsysteme, } \\
\text { Fernwärmernetze } \\
\text { - Erschwernis für neue } \\
\text { (betriebswirtschaftlich } \\
\text { teurere) Technik } \\
\text { - Nachteil für Einspar- } \\
\text { technik } \\
\text { - Widersprüchlichkeit bei } \\
\text { ökologischen Steuern }\end{array}$ & 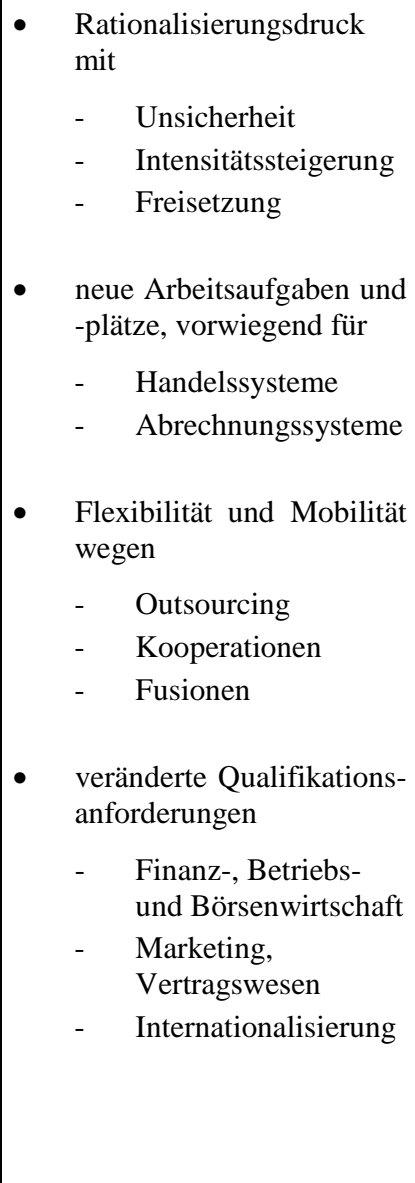 & \begin{tabular}{|ll} 
- & $\begin{array}{l}\text { Energiebörse, } \\
\text { e-Commerce }\end{array}$ \\
- & multinationale \\
- & regionzerne \\
& für Beschäftigung, \\
& Wertschöpfung etc. \\
- & Versorgungssicherheit \\
- & verfügbares Einkom- \\
men privater Haushalte & wegen Veränderung \\
der Höhe, Differenzie- \\
rung und Struktur der \\
Strompreise
\end{tabular} \\
\hline
\end{tabular}


Dass die sozialen Implikationen grundlegend und nachhaltig sein können, ergibt sich nicht nur aus der Freisetzung von vielen Beschäftigten. Letztere beträgt z. B. bei den Stadtwerken in den $1 \frac{1}{2} 2$ Jahren seit April 1999 über $13 \%$ (Reduktion von 150 Tsd. auf 130 Tsd. Beschäftigte). Durch die vollständige Ausrichtung der Stromwirtschaft auf kommerzielle Ziele werden zugleich stark veränderte Anforderungen an die Qualifikation der Weiterbeschäftigten gestellt. Die Tendenz geht dahin, zumal der Anteil der Eigenerzeugung der Lieferanten zugunsten des billigen Einkaufs und des Stromhandels sinkt, immer weniger Wissen und Erfahrung ingenieurtechnischer, dafür immer mehr kaufmännischer, juristischer und händlerischer Art zu verlangen. Hinzu kommt, dass einerseits vielfach neue Geschäftsfelder (wie bspw. Kundenwerbung, Verbraucherberatung, Paketangebote mit Versicherungen, Kreditvergabe und internationaler Handel) im Sinne der Entwicklung zu Multi-Utilities aufgebaut und personell bedient werden müssen. Andererseits ist mit der Privatisierung und zunehmenden Firmenkooperation/-fusion eine Tendenz der Internationalisierung mit entsprechenden Personalanforderungen unverkennbar.

In diesem Zusammenhang muss auch betont werden, dass sich die veränderten Rahmenbedingungen nicht nur in der Elektrizitätswirtschaft selbst auswirken. In die vorgelagerten Kooperationsstufen, z. B. bei der Herstellung von Energieanlagen (Turbinen, Kessel, Generatoren usw.) wurde zwangsläufig der Wettbewerb ebenfalls eingeführt bzw. intensiviert, nicht zuletzt wegen der veränderten Nachfragesituation aufgrund neuer Technik-/ Technologiekonfigurationen. Konkret verdeutlicht sich das z. B. im Gas- gegenüber dem Atomkraftwerksgeschäft und in der Expansion der Zulieferer für die moderne Netztechnik, vor allem zur Messung und Abrechnung der wechselnden Fremddurchleitungen.

Unter dem Druck des Wettbewerbs und der bis zu $30 \%$ sinkenden Gewinnmargen im Stromsektor entstehen auch Implikationen für jene Geschäftsfelder, die bisher im Rahmen von Quersubventionen/Querverbünden vom gewinnintensiven Stromverkauf profitieren konnten. Dazu zählen hauptsächlich die in der Regel wenig renditeträchtige bzw. verlustgefährdete
Wärmewirtschaft und der Nahverkehr bei Stadtwerken.

Zugleich ist darauf hinzuweisen, dass die mit dem Wettbewerb auf dem Strommarkt einher gehende Preissenkung für bestimmte (durchaus zukunftsfähige) Technologien/Technikentwicklungen negative Auswirkungen mit sich bringen kann. Das betrifft insbesondere die Energieeinsparungen und die Nutzung regenerativer Energien (wie Wind-, Wasserkraft sowie Biomasse). Ihre Technologien und Techniken sind zur Zeit vielfach im betriebswirtschaftlichen Sinn noch nicht rentabel. Gegenüber den herkömmlichen, bereits abgeschriebenen Energie-Großanlagen können sie sich in der Regel bei sinkenden Strompreisen auf dem gesättigten Strommarkt kaum etablieren und ausdehnen.

Da sie andererseits aber energie- und umweltpolitisch erhebliche Vorteile aufweisen, die sich erst in einer komplexen gesamtwirtschaftlichen und nicht nur betriebswirtschaftlichen Bewertung reflektieren, erscheint hierfür ein staatlicher Schutz vor dem Marktautomatismus gerechtfertigt.

\section{Veränderungen auf der Nachfrage- seite}

Nachfrageseitig kommt es insbesondere wegen erheblicher Preisreduzierungen zu Veränderungen. Für größere Verbraucher der Wirtschaft (Sondervertragskunden) fielen bisher die Strompreise durchschnittlich um etwa $30 \%$, für die privaten Haushalte und Gewerbekunden (Tarifkunden) konnten Preisreduzierungen bis etwa $10 \%$ erreicht werden. Diese veränderte Bewertung beeinflusst natürlich Entwicklungsoptionen und das individuelle Verbraucherverhalten der Stromkunden. Während z. B. Elektrotechnologien eine Aufwertung erfahren, lohnen sich Strom- und Energieeinsparungen bei niedrigen Preisen immer weniger. Das ist besonders für Technologien/Anlagen an der Schwelle der Wirtschaftlichkeit bedeutsam.

Positive Entwicklungsimpulse können sich so z. B. in modernen Niedrigenergiehäusern für die elektrische Heizung sowie für den Einsatz von Wärmepumpen ergeben, vor allem im Vergleich zum Fernwärme-Anschluss. Das verlangt dann auch die Neubewertung der Technikfolgen. Stehen doch die Elektro- und 
Wärmepumpenheizungen gegenüber dem Fernwärmeverbund bspw. mehr oder weniger für die Individualisierung der Verbraucherwünsche und die relative Unabhängigkeit des Kunden von großflächigen Systemlösungen.

Die Einführung von Wettbewerb bewirkt außerdem auf der Nachfragseite, dass sich bei den Kunden erstmals eine Bereitschaft zum Lieferantenwechsel mit all seinen Konsequenzen herausbildet. Die Akzeptanz der Stromlieferung wird nun - in Erweiterung zur ehemaligen einheitlichen, sicheren und wenig variablen Versorgung - neu zu begreifen sein. Wer jetzt den Lieferanten wechselt, gewinnt durchaus Preisvorteile, muss aber auch mit kaum vergleichbaren, wenig transparenten Differenzierungen, Unsicherheiten und Flexibilitäten (im Preis, in der Betreuung, im Service etc.) rechnen.

\section{Schlussfolgerungen für TA-Analysen}

Technikfolgenabschätzung befasst sich generell mit den gesellschaftlichen Folgen technologischer Entwicklungen. Ohne Zweifel spielt dabei die Energieversorgung - namentlich wegen ihrer nachhaltigen Wirkung auf das Wirtschafts-, Umwelt- und Sozialgefüge - eine zentrale Rolle. Bei der Erforschung und Bewertung ihrer Folgen und Nebenwirkungen wird die Analyse der grundlegenden Veränderungen institutioneller Rahmenbedingungen, ihre Veränderungen und ihre Gestaltungsmöglichkeiten an Bedeutung zunehmen. Letztere zählen zu den entscheidenden Ausgangs- und Realisierungsbedingungen technischer Entwicklungen und ihrer Umsetzung, wie am Beispiel der Liberalisierung der Strommärkte ausgeführt.

Der gegenwärtige Trend zur Liberalisierung der Wirtschaft - in Einheit mit Deregulierung und Privatisierung - stellt die TA vor große Herausforderungen. Die Einbeziehung fundamentaler Veränderungen im Bedingungsgefüge der Technikanwendungen ermöglicht es, damit verbundene gesellschaftliche Folgen frühzeitig $\mathrm{zu}$ antizipieren und negative Entwicklungen zu erkennen.

\section{Kontakt}

Prof. Dr. Martin Weisheimer Institut für Wirtschaftsforschung Halle Postfach 1103 61, D-06017 Halle (Saale)

Tel.: + 49 (0) 345 / 7753 - 708

Fax: + 49 (0) 345 / 7753 - 825

E-Mail: wsh@iwh-halle.de 